\title{
BIDIMENSIONALISMO EPISTÉMICO Y EL ESPACIO ONTOLÓGICO MODAL
}

\author{
José Tomás Alvarado Marambio \\ Instituto de Filosofía \\ Pontificia Universidad Católica de Valparaíso \\ jose.alvarado.m@ucv.cl
}

\begin{abstract}
RESUMEN: Este trabajo presenta las líneas centrales del bidimensionalismo epistémico defendido recientemente por David Chalmers, y considera, en particular, las motivaciones a las que la semántica debería servir en la conexión entre modalidad metafísica y epistémica. Después de esta presentación, se indican tres dificultades: (i) no se ha diferenciado suficientemente de las semánticas bidimensionales contextuales tradicionales; (ii) la noción de justificación a priori sobre la que se ha desarrollado requiere urgentemente mayor precisión; y (iii) los defensores del esquema han olvidado completamente los casos de "contingencias fuertes".
\end{abstract}

PALABRAS CLAVE: bidimensionalismo, modalidad, a priori, semántica

SUMMARY: This work presents the main lines of epistemic two-dimensionalism recently defended by David Chalmers, discussing, in particular, the motivations that semantics is supposed to serve in the connection between metaphysical modality and epistemic modality. After presenting the two-dimensional epistemic semantics, three difficulties are leveled against it: (i) it is insufficiently distinguished from the traditional contextual bi-dimensional semantics; (ii) the notion of a priori justification on which it is constructed is badly requiring more precision; and (iii) the defenders of the semantics have completely despised the cases of "strong contingencies".

KEY WORDS: two-dimensionalism, modality, a priori, semantics

En una serie de escritos, David Chalmers (1996, pp. 83-128; 2006) y Frank Jackson (1998, pp. 1-86) han propuesto un ambicioso programa en el que la utilización de una semántica bidimensional permitiría resolver variados problemas filosóficos mediante una reflexión conceptual a priori. Kripke ha sostenido que hay enunciados necesarios a posteriori (cfr. 1980, pp. 38-39), por lo que pareciera que la estructura modal del mundo ya no es algo que pueda ser descubierto mediante el análisis a priori de los filósofos. Chalmers y Jackson, en cambio, sostienen que los enunciados necesarios a posteriori también pueden ser justificados por un procedimiento que sólo conlleva la reflexión sobre el contenido de nuestros conceptos. Para esto distinguen entre lo que denominan una "intensión primaria" y una "intensión secundaria". La intensión primaria sería un componente "interno" que operaría como un "selector" de algún aspecto del mundo. La intensión secundaria, en cambio, sería el resultante de esa selección. 
En efecto, si es necesario que el agua sea $\mathrm{H}_{2} \mathrm{O}$, por ejemplo, se debe a que, dado como está constituido el mundo actual, cierta intensión primaria de "agua" ha seleccionado una sustancia compuesta por moléculas de $\mathrm{H}_{2} \mathrm{O}$ y, por lo tanto, se ha configurado una intensión secundaria de agua como $\mathrm{H}_{2} \mathrm{O}$. La intensión primaria es un componente que resultaría perfectamente transparente para la reflexión a priori, mientras que la intensión secundaria depende de cómo esté constituido el mundo y, por lo tanto, en algún sentido, no se encuentra "en la cabeza" de los hablantes. La misma intensión primaria podría haber seleccionado una sustancia diferente como $X Y Z$ y, por consiguiente, desde la perspectiva de las intensiones primarias podría haber sucedido que el agua fuese $X Y Z$ y no $\mathrm{H}_{2} \mathrm{O}$. Esto se sigue simplemente de la reflexión sobre el contenido de la intensión primaria del concepto agua y de la reflexión sobre los diferentes "escenarios" en que esa intensión primaria podría seleccionar una intensión secundaria. Esta propuesta ha generado una fuerte controversia, como es natural ( $c f r$. por ejemplo, Soames 2005). Este artículo pretende indicar algunas dificultades en la propuesta de bidimensionalismo epistémico presentada por Chalmers.

Usualmente, en efecto, se ha considerado que las semánticas bidimensionales especifican la forma en que el contenido de los enunciados depende de factores contextuales en los que los enunciados son proferidos (o pensados). ${ }^{l}$ Chalmers quiere distanciar su propuesta epistémica de las formas de bidimensionalismo contextual y señala:

El problema es que la aprioridad y ser-verdadera-cuando-se-usa [una oración] son nociones fundamentalmente diferentes. La primera opera sobre un elemento epistémico o racional, pero la segunda no opera sobre un elemento semejante. La segunda noción opera sobre un elemento metalingüístico, pero la primera no opera sobre un elemento semejante. (2006, p. 70)

En una interpretación contextual, por ejemplo, la oración "Yo profiero ahora una oración" ha de resultar necesaria, pues en cualquier contexto que se emplee esa oración tendrá que ser verdadera, aun cuando parezca un hecho obviamente contingente el que alguien profiera o no una oración determinada. Chalmers quiere desarrollar un espacio modal directamente ligado a lo que puede ser determinado mediante una reflexión a priori de manera que quede restablecida

\footnotetext{
${ }^{1}$ Un ejemplo característico es el tratamiento que Kaplan hace de las expresiones deícticas. Cfr. Kaplan 1990.
} 
la que llama, "conexión áurea" entre necesidad, significado y aprioridad. Según ésta, un enunciado es necesario si y sólo si tiene una justificación a priori, justificación que ha de depender del contenido de las intensiones primarias de los términos que aparecen en el enunciado necesario ( $c f r$. Chalmers 2006, pp. 55-59). La semántica bidimensional epistémica se define sobre un espacio modal según el cual:

(1) $\diamond^{e} S \leftrightarrow(S$ no es refutable a priori $)$.

Aquí " $\diamond$ " es el operador de posibilidad epistémica. De la misma manera se establece que:

(2) $\square^{e} S \leftrightarrow(S$ es justificable a priori).

El contenido de los enunciados tal que éstos resulten verdaderos o falsos en los diferentes escenarios es su intensión primaria. Es fundamental aquí la noción de justificación a priori. Chalmers caracteriza un "pensamiento a priori" como aquel que "puede ser justificado de manera concluyente y no experiencial sobre la base de reflexión racional ideal" (2006, p. 98). No se especifica ulteriormente qué sea una reflexión racional "ideal", pero conlleva poner entre paréntesis las limitaciones cognitivas de algún sujeto racional en particular.

El objetivo de Chalmers es justificar que la modalidad epistémica - ligada a la semántica bidimensional indicada - es la modalidad metafísica, esto es:

(3) $\forall S\left[\diamond S \leftrightarrow \diamond^{e} S\right]$

Chalmers no ha desarrollado una argumentación detallada para justificar esta tesis. Se trata simplemente de un ambicioso programa de investigación para sustituir la modalidad metafísica por la modalidad epistémica. Por un lado, Chalmers ha rechazado contraejemplos a la tesis (3) en que un estado de cosas sea necesario y, sin embargo, pueda concebirse que sea falso. Estos contraejemplos son denominados "necesidades fuertes". Esto es, una necesidad fuerte es un enunciado que resulta necesario pero que, sin embargo, posee una negación concebible. Los ejemplos característicos en los que está pensando Chalmers son la existencia de Dios o las identificaciones psicofísicas. Chalmers destaca que no hay necesidades fuertes que hayan sido justificadas y que, además: 
Podríamos argumentar con más plausibilidad en el sentido inverso: en cada uno de esos casos, la conexión entre ser algo concebible y ser algo posible, no rota en otros casos, ofrece un argumento contra las suposiciones en cuestión. En todo caso, no hay contraejemplos claros aquí contra la tesis. (2002, p. 192)

El programa debería conectar las nociones modales con el "dominio racional" (2002, p. 192). Por supuesto, Chalmers no posee un argumento mediante el cual se efectúe esa conexión, pero confía en que se puede llegar a desarrollar uno. Se trata de un programa de investigación.

Puede entonces argumentarse que este espacio de mundos es suficiente para explicar todos los fenómenos modales para los que tenemos razones en creer. Este espacio analizará materias racionales y psicológicas como el pensamiento contrafáctico, la inferencia racional y los contenidos del pensamiento y el lenguaje, tal como cualquier otro espacio modal puede hacerlo. Y con la ayuda de la evaluación semántica bidimensional, este espacio puede acomodar fenómenos modales "metafísicos" como la distinción concepto/propiedad, las necesidades a posteriori, etc. [...] De modo que el espacio modal, junto con el análisis conceptual y hechos no modales, nos da todo, siempre que este espacio modal esté constitutivamente conectado con el dominio racional. (Chalmers 2002, p. 193)

En lo que sigue se presentarán tres dificultades de principio contra este programa de investigación y contra el esquema bidimensional epistémico que le presta sustento. Tal como habrá ocasión de observarlo, a pesar de los esfuerzos recientes de los propugnadores del bidimensionalismo ambicioso, subsisten poderosos motivos para pensar que no se ha desarrollado una concepción alternativa suficientemente plausible que pueda oponerse a las concepciones semánticas (teoría de la referencia directa) y metafísicas (postulación de un espacio modal ontológico objetivo) ${ }^{2}$ prevalecientes. Estas dificultades son: (i) que no se ha conseguido diferenciar con suficiente precisión y de un modo aceptable el bidimensionalismo epistémico de las diversas formas de bidimensionalismo contextual; (ii) que la noción de justificación a priori es problemática, al menos para la constitución de un espacio modal epistémico tal como se ha pretendido; y (iii) que el

\footnotetext{
${ }^{2}$ Esto es, la idea de que lo necesario y lo posible no son simplemente un producto de nuestra imaginación o de nuestras capacidades de concebir, sino que constituyen un rasgo objetivo del mundo que debemos tratar de descubrir como cualquier otro.
} 
hiato entre modalidad epistémica y modalidad metafísica es mucho mayor de lo que Chalmers parece suponer. A continuación se verán estas dificultades en orden.

\section{El bidimensionalismo epistémico también es contextual}

El bidimensionalismo epistémico se ha presentado en contraste con las formas contextuales de bidimensionalismo. La idea general del esquema bidimensional epistémico es que refleja lo que puede ser justificado a priori por un sujeto racional. Dado lo justificable a priori y, correlativamente, lo que no puede refutarse a priori (correspondientes, respectivamente, a la necesidad y a la posibilidad), se puede definir cierta totalidad de escenarios epistémicos, esto es, descripciones máximamente específicas sobre cómo podría estar constituido el mundo dado lo que es justificable a priori. La necesidad es aquí la verdad en todos los escenarios y la posibilidad es la verdad en al menos uno de los escenarios. ¿Qué son aquí las intensiones primarias y secundarias? En general, la intensión de una oración es el conjunto de todos los escenarios en los que es verdadera (o la secuencia de valores de verdad en los diferentes escenarios de esa oración). Con las modificaciones del caso, este criterio permite definir también intensiones para términos (nombres y predicados) que puedan ocurrir en las oraciones. Tal como se presenta la cuestión, el punto de partida para asignar intensiones a los términos de un lenguaje y a las oraciones que se puedan formar por medio de tales intensiones es la asignación de valores de verdad a priori en diferentes escenarios. Dada la distribución de valores de verdad en los distintos escenarios puede decidirse si dos oraciones son o no equivalentes materialmente a priori. Los hechos sobre qué intensiones existen quedan fijados, entonces, mediante la determinación de qué equivalencias a priori se dan (o no se dan). ${ }^{3}$ Esta asignación, siendo a priori, tiene que efectuarla algún sujeto mediante una reflexión racional idealizada (recuérdese que ésta es la definición de justificación a priori).

\footnotetext{
${ }^{3}$ Otro problema que no se va a tratar aquí es que la asignación de valores de verdad para todas las oraciones de un lenguaje en todos los escenarios (el equivalente epistémico de un mundo posible) no es suficiente para fijar las referencias de los términos de ese lenguaje $L$. Esa asignación es compatible con permutaciones que varíen de manera arbitraria cualquier extensión dada de los términos que se haya estipulado. Esto es lo que muestra el famoso argumento de teoría de modelos de Putnam ( $c f r$. especialmente la formulación en Putnam 1981, pp. 22-48, 217218). Por lo tanto, Chalmers debería estipular un mecanismo independiente para la asignación de intensiones a nombres y predicados del que dependieran luego las asignaciones de valores de verdad para las oraciones.
} 
Lo que resulta justificable a priori varía entre distintos sujetos y en el tiempo para un mismo sujeto y, correlativamente, el espacio modal epistémico definido y las intensiones que de ahí resultan son igualmente variables. Esto puede dejarse aquí a un lado. Supóngase un instante de tiempo fijo y un sujeto racional determinado. En relación con este sujeto racional y este instante de tiempo, hay hechos determinados sobre lo que es o no justificable a priori ${ }^{4} \mathrm{y}$, luego, se puede postular el espacio epistémico en cuestión sobre este tiempo y este sujeto. En principio, lo que diferencia al enfoque epistémico de un esquema bidimensional respecto de algún enfoque contextual es que representa cierto contenido de información poseído por un sujeto racional y no la dependencia de la proferencia de una oración (o del acto intencional de juzgar algo) respecto del contexto en el que ese acto de proferencia ocurre. Chalmers quiere desligar el esquema bidimensional de las interpretaciones en las que resultan necesarias oraciones como "digo algo ahora". Una intensión primaria epistémica está asociada a la información poseída a priori por un sujeto racional que puede seleccionar una intensión secundaria con independencia de que existan o no actos de habla de aseveración de la oración en cuestión (o bien actos intencionales en los que se juzga como verdadero un contenido).

El problema, sin embargo, es que la remisión contextual reaparece nuevamente en una interpretación epistémica. Algo es justificable (o, correlativamente, no es refutable) a priori para alguien. Los "escenarios" epistémicos son tratados por Chalmers, en efecto, o bien como descripciones canónicas, o como mundos posibles centrados en los que se refleja la perspectiva epistémica que tiene un sujeto racional en un mundo posible en un instante ( $c f r$. Chalmers 2006, pp. 75107). Pues, bien, considérese la intensión que resulta de la siguiente oración:

(4) Un sujeto racional existe ahora.

$\mathrm{O}$, si se quiere destacar que la oración documenta una creencia de se:

(5) Yo (a saber, un sujeto racional) existo ahora.

Esta tesis cartesiana resulta necesaria en cualquier semántica bidimensional epistémica, pero es obvio que refleja una proposición

\footnotetext{
${ }^{4}$ ¿Los hay? Tal vez no, pero por el momento puede obviarse esta cuestión. Supóngase simplemente que cada oración de un lenguaje $L$ es determinadamente justificada o es determinadamente no justificada mediante la reflexión racional idealizada.
} 
contingente pues yo soy un ente contingente. De hecho, en cualquier escenario epistémico que se considere debe haber algún sujeto racional para el que es dada una perspectiva epistémica sobre lo que acaece en ella (y en los escenarios alternativos: recuérdese que la perspectiva epistémica define un espacio modal bidimensional). Si Chalmers considera inaceptable o, al menos, inconveniente que enunciados sobre la ocurrencia de ciertos actos de habla o sobre la ocurrencia de ciertos actos intencionales resulten necesarios, entonces también debería resultar inaceptable o, al menos, inconveniente la existencia necesaria de algún sujeto racional dotado de cierta perspectiva epistémica.

El esquema bidimensional epistémico estaba diseñado a la medida para satisfacer la "tesis central", esto es, que una oración es necesaria si y sólo si es a priori. Se trata de la forma de restablecer la conexión áurea entre significado, aprioridad y necesidad destruida después de Kripke. Esta conexión entre significado, aprioridad y necesidad es una de las motivaciones fundamentales para preferir la interpretación epistémica, ligada a lo que puede o no justificarse a priori, por sobre las diversas interpretaciones contextuales. No debería resultar "necesario" ni "a priori" que alguien diga algo o que alguien piense algo. Pero sucede que en la forma de comprender el esquema para restablecer la conexión áurea aparece como "a priori" y "necesaria" la existencia de un pensador. En principio, el conocimiento que tiene cada uno de nosotros sobre sus propios estados mentales y sobre el hecho de ser un pensador racional es una materia de introspección, pero Chalmers expresamente rechaza la introspección como una forma de justificación a priori ( $c f r$. Chalmers 2006, p. 99). Considérese, además, que el proyecto de fondo consiste en reemplazar la modalidad metafísica por la modalidad epistémica. Hay una fuerte intuición aquí de que la existencia de sujetos racionales es contingente y esa intuición, por lo menos, no es capturada en el espacio modal epistémico.

Chalmers podría quizá apelar a intensiones epistémicas "extendidas". Una intensión sistémica "extendida" de una oración es la indicación del contenido de información que sería comprendido por un sujeto racional $S$ si es que $S$ existiese en un escenario, aunque de hecho $S$ no exista en ese escenario. En este caso, la existencia de un pensador no resultaría necesaria a priori. El problema con este procedimiento, sin embargo, es que el mismo Chalmers lo rechaza por ser confuso y muy difícil de precisar satisfactoriamente para el caso de intensiones contextuales (cfr. Chalmers 2006, pp. 72-73). Las intensiones en cuestión y el entero espacio modal definido dependerían de condicionales contrafácticos cuyas condiciones de verdad 
deberían estar claras antes de constituir el espacio modal, cuando usualmente es el aparato modal el que permite asignar esas condiciones de verdad. Y Chalmers pretende explicar todas las modalidades mediante el espacio epistémico.

Por supuesto, puede seguirse sosteniendo que el espacio modal epistémico y las intensiones (primarias y secundarias) ligadas a él cumplen funciones teóricas fructíferas para la explicación de ciertos fenómenos como, tal vez, el contenido del pensamiento y de otros estados mentales, pero ciertamente no parece una herramienta apropiada como la llave de bóveda de toda nuestra comprensión de las nociones modales. Al menos, no sin ulterior argumentación y sin que estas dificultades sean resueltas satisfactoriamente.

\section{2. ¿Qué es justificable a priori?}

Tal como se ha podido apreciar, el espacio modal epistémico está constituido por lo que puede ser justificado a priori (por un sujeto racional en un escenario). Una oración $S$ es justificable a priori, en la definición dada, si y sólo si tuviera que ser aceptada después de una reflexión racional idealizada. Tal como se vio también, Chalmers se niega a precisar demasiado qué debe entenderse aquí por una reflexión "idealizada" (para no "multiplicar las nociones modales"), salvo la indicación de que por la idealización deben ponerse entre paréntesis las limitaciones cognitivas contingentes que podamos tener. Es obvio, sin embargo, que la importancia que se ha otorgado a este concepto obliga a examinar con sumo cuidado su comportamiento. Después de todo, Chalmers pretende que el espacio modal completo, la dilucidación de cada una de nuestras nociones modales, esté - finalmente - pendiendo de qué haya de ser una justificación a priori.

De hecho, los seres humanos cometemos toda clase de errores en materias formales tal como lo hacemos en materias empíricas. Así como podemos equivocarnos al aceptar una percepción sensible engañosa, también podemos equivocarnos al realizar un cálculo, al hacer y evaluar una prueba o, sencillamente, al juzgar sobre la aplicación de un concepto en un caso específico. Lo que resulta de nuestra reflexión racional es muchas veces erróneo. Si el espacio modal se hace depender de lo que pueda inclinarse a aceptar un ser humano por reflexión racional, entonces el espacio modal sería incoherente. La reflexión racional ha considerado perfectamente aceptable una tesis como el axioma V del Grundgesetze der Arithmetik, pero Russell 
mostró que era inconsistente, por ejemplo. Se supone que la "idealización" va a resolver estas dificultades. Aunque de hecho se cometan errores, olvidos y desatenciones, es posible que no se cometan tales errores y, en principio, podemos quedarnos con ese "mundo posible". 5 Por supuesto, hay muchos otros "mundos posibles" con más errores, olvidos y desatenciones. De algún modo, la justificación de $S$ por reflexión racional idealizada queda fijada por lo que aceptaría un sujeto pensando por reflexión racional en aquellos mundos posibles "marcados" o "fijados".

La pregunta obvia aquí, sin embargo, es ¿qué criterios o motivos existen para fijar la justificación a priori sólo por lo que acaece en esos mundos posibles "fijados"? Intuitivamente se han "fijado" esos mundos posibles por el hecho de que en ellos la reflexión racional entrega la respuesta "correcta". Pero, ¿según qué parámetros es "correcta" la respuesta de la reflexión racional? No sirve, desde luego, atender a la respuesta que entregaría la reflexión racional en tales y cuales mundos posibles, pues es la corrección de esas respuestas judicativas lo que se pretende discriminar en lo tocante a su corrección o incorrección. Si hay algún criterio independiente para saber cuáles son las respuestas correctas de la reflexión racional (aquellas que contarán como "idealizadas"), éste debe depender de ciertos hechos sobre qué se deduce lógicamente de qué. Estos hechos son necesarios, naturalmente. La corrección o incorrección de una reflexión racional es el hecho de que se atiene a las relaciones de consecuencia lógica realmente existentes entre diferentes proposiciones, y considera coherente lo que es coherente y considera incoherente lo que es incoherente. Entonces resulta que la modalidad epistémica definida por Chalmers sólo puede ser coherente si es que depende de una forma de modalidad más básica. Una forma de modalidad que, por lo menos, podría ser caracterizada como modalidad lógica, si es que no se quiere admitir sencillamente que se trata de una modalidad metafísica.

\footnotetext{
${ }^{5}$ Que en este punto se deba recurrir a un mundo posible es, naturalmente, otro problema que Chalmers ha querido escamotear dejando la noción de "idealización" como primitivo. Es obvio, sin embargo, que se necesita explicar cómo es que opera la "idealización" en este respecto por la importancia sistemática que se le asigna. Como aquí la aprioridad es utilizada para definir los escenarios, no se pueden utilizar escenarios para definir qué es una justificación a priori. ¿Qué son estos mundos posibles, entonces? Pareciera que son sencillamente los viejos mundos posibles metafísicos. Este problema no será discutido con más extensión aquí porque las dificultades que se indicarán son de por sí suficientes para mostrar deficiencias importantes en el esquema propuesto por Chalmers.
} 
Es obvio que Chalmers ha intuido estas dificultades cuando se ha resistido a explicar o analizar más la noción de "reflexión racional idealizada" y ha preferido dejarla como un primitivo. El problema, sin embargo, es que es obvio también que se trata de explicaciones indispensables si es que todo el espacio modal que está siendo definido depende de la justificación a priori y si, todavía más, Chalmers pretende llegar a mostrar que todas nuestras nociones modales, sin residuo, podrán ser tratadas con la modalidad epistémica. Esta explicación es urgente cuando, tomada la noción sin prevenciones, resultan incoherencias. Debe haber motivos no ad hoc para rechazar los resultados incoherentes (como la aceptación por reflexión racional del axioma V del Grundgesetze), pero es difícil determinar cuáles pueden ser esos criterios si no es una forma de modalidad más básica que aquella que se pretende introducir aquí. Pareciera, por lo tanto, que el espacio modal epistémico sobre el que Chalmers quiere definir su esquema bidimensional es o bien dependiente de una modalidad lógica o metafísica que tiene un carácter fundamental, o se trata de una noción inconsistente. En el primer caso, el programa ambicioso de reducir lo posible a lo concebible (la modalidad metafísica a la modalidad epistémica) fue un fracaso. En el segundo caso, no podemos tomar en serio el espacio modal epistémico.

Una cuestión adicional tiene que ver con el alcance de la justificación racional idealizada. En principio, aquello que puede ser justificado a priori, según la definición de esta noción, es variable en el tiempo y para distintos sujetos. Considérese, por ejemplo, el caso del último teorema de Fermat en el año 1900. ¿Era o no epistémicamente necesario entonces? ¿El hecho de que no existiese una demostración de ese teorema se debía simplemente a limitaciones contingentes que podemos desechar en una idealización? No había errores de cálculo ni graves desatenciones en 1900 por parte de la comunidad matemática, pero sencillamente no se había llegado al nivel de desarrollo suficiente para la demostración. Del mismo modo uno puede preguntarse si es que la conjetura de Goldbach o la Hipótesis del Continuo de Cantor son o no ahora epistémicamente necesarias. ¿Qué veredicto entrega respecto de estos casos la reflexión racional idealizada? El problema que se destacó anteriormente sobre la noción de aprioridad tenía que ver con la exclusión de errores mediante algún criterio independiente. La cuestión que se trata aquí tiene que ver con la extensión de las capacidades epistémicas para determinar el valor de verdad de un enunciado mediante reflexión racional "idealizada". ¿Hacia dónde se deben extender las capacidades epistémicas en cuestión? ¿Debe tomarse en consideración sólo lo que seres racionales como nosotros 
podemos procesar cognitivamente? ¿Por qué no considerar las capacidades cognitivas de seres racionales mucho más perspicaces que nosotros, con más capacidad de procesamiento de información? Es razonable sostener que nosotros, sin cometer errores, no estábamos en condiciones de probar el teorema de Fermat en 1900, pero que tal vez una comunidad matemática alienígena sí estaba en condiciones de hacerlo. Tal vez ahora, por ejemplo, una comunidad de ángeles sí pueda probar la conjetura de Goldbach, aunque nosotros no poseamos esa prueba por reflexión racional "idealizada" (en el sentido de excluir los errores y las desatenciones). Alguien podría entonces sostener, en este caso, que no sabemos que existan comunidades de alienígenas racionales ni de ángeles, pero esto no importaría para los propósitos que se tienen en mira. Después de todo se trata de una "idealización" y si de idealizaciones se trata bien podríamos apelar a lo que pueden determinar por reflexión racional entidades posibles, como alienígenas, ángeles o Dios.

Resulta, por lo tanto, que la noción de "reflexión racional idealizada" requiere urgentemente mucha mayor precisión. No sólo existe el problema apuntado con anterioridad sobre cómo fijar los resultados posibles correctos diferenciándolos de los incorrectos, sino también existe el problema de determinar qué clase de capacidades cognitivas deberán ser el patrón por el que habrá de fijarse el rango de verdades justificables a priori, esto es, por reflexión racional idealizada. En el peor de los casos, la noción colapsa en la mera verdad si es que las capacidades cognitivas de procesamiento de información son máximas. Después de todo, Dios es omnisciente y él, mediante una reflexión racional sobre el contenido de su propia esencia, conoce todas las verdades. Sería, sin embargo, un despropósito recurrir a capacidades cognitivas tan exorbitantes.

\section{Modalidad epistémica y modalidad metafísica}

Tal como se ha indicado anteriormente, el programa ambicioso de Jackson y Chalmers consiste en reivindicar la importancia del análisis conceptual a priori para la decisión de problemas metafísicos, por ejemplo, decidir sobre la reducción de cierto nivel de entidades a otro mediante la determinación de ciertas relaciones de implicación (implicación material a priori) entre conjuntos de enunciados. Con este fin, Chalmers ha desarrollado un espacio modal epistémico en el que pueden ser definidas intensiones primarias y secundarias. El proyecto ambicioso de Chalmers es mostrar que todas las funciones teóricas que han sido asignadas a la modalidad metafísica pueden 
cumplirse satisfactoriamente mediante la modalidad epistémica. Para esto se necesita justificar la tesis (3):

(3) $\forall S\left[\diamond S \leftrightarrow \diamond^{e} S\right]$

Esto es, una oración o enunciado $S$ es posible metafísicamente si y sólo si $S$ es epistémicamente posible, esto es, $S$ es metafísicamente posible si y sólo si $S$ no es refutable a priori. Lo que de hecho ha discutido Chalmers es la tesis:

(6) $\forall S\left[\diamond^{e} S \rightarrow \diamond S\right]$

Un contraejemplo de esta tesis (6) sería un estado de cosas (o una oración que se hace verdadera por tal estado de cosas) que sea epistémicamente posible pero que no sea metafísicamente posible. La contrapositiva de (6) es que si algo es imposible metafísicamente, entonces es imposible epistémicamente. De esto se sigue que si algo es metafísicamente necesario, entonces es epistémicamente necesario. ${ }^{6}$ Por esto, Chalmers discute de manera expresa los casos de enunciados metafísicamente necesarios que fuesen epistémicamente contingentes como posibles contraejemplos. Si se quiere, junto con la tesis (6) debe ponerse explícitamente su par: ${ }^{7}$

(7) $\forall S\left[\square S \rightarrow \square^{e} S\right]$

La discusión de los condicionales (6) y (7), sin embargo, sólo refleja una mitad de la tarea necesaria para la justificación del bicondicional (3) en el que la modalidad metafísica sea capturada por la modalidad epistémica. Restan por considerar las tesis:

(8) $\forall S\left[\diamond S \rightarrow \diamond^{e} S\right]$

(9) $\forall S\left[\square^{e} S \rightarrow \square S\right]$

Estos otros dos condicionales cuantificados reflejan la segunda mitad de la tarea requerida para justificar el principio (3). En primer

\footnotetext{
${ }^{6}$ La contrapositiva de (6) es $\left(\forall S\left[\neg \diamond S \rightarrow \neg \diamond^{e} S\right]\right)$. Si se transforman las expresiones " $\neg \diamond$ " en " $\square \neg$ " resulta $\left(\forall S\left[\square \neg S \rightarrow \square \square^{e} \neg S\right]\right)$. Ahora se sustituye " $\neg S$ " por " $S$ " y queda $\left(\forall S\left[\square S \rightarrow \square^{e} S\right]\right)$.

${ }^{7}$ En efecto, de la tesis (3) $\left(\forall S\left[\diamond S \leftrightarrow \diamond^{e} S\right]\right)$ se sigue que $\forall S\left[\neg \diamond S \leftrightarrow \neg \diamond^{e} S\right]$. Transformando " $\neg \diamond$ " por " $\square \neg$ " resulta $\left(\forall S\left[\square \neg S \leftrightarrow \square \square^{e} \neg S\right]\right)$. Luego, sustituyendo " $\neg S$ " por " $S$ " queda $(\forall S[\square S \leftrightarrow \square$ ' $S])$. En este bicondicional cuantificado también se pueden inferir condicionales de derecha a izquierda y de izquierda a derecha.
} 
lugar, el principio (8) enuncia que si algo es metafísicamente posible, entonces es epistémicamente posible. Un contraejemplo de este principio es un estado de cosas (o enunciado) metafísicamente posible pero epistémicamente imposible. Por otro lado, el principio equivalente (9) enuncia que si un estado de cosas (o enunciado) es epistémicamente necesario, entonces es metafísicamente necesario. Un contraejemplo de este principio sería el caso de estados de cosas epistémicamente necesarios pero metafísicamente contingentes. Casos de esta especie podrían ser denominados "contingencias fuertes", tal como Chalmers ha denominado "necesidades fuertes" a los enunciados metafísicamente necesarios pero epistémicamente contingentes.

El problema para el planteamiento de Chalmers es que existen casos de contingencias fuertes o, al menos, parecen existir, y estos casos presentan muchas más dificultades que los casos de necesidades fuertes, por muy poco convincente que pueda parecer el modo displicente en que Chalmers ha rechazado tales contraejemplos. Considérese este enunciado:

(10) La geometría del espacio-tiempo es curva

Este enunciado es, en principio, una verdad empírica sobre el mundo que habitamos, una verdad contingente que podría haber sido falsa de acuerdo con la teoría de la relatividad general. Por supuesto, la posibilidad de que el mundo posea una geometría del espaciotiempo euclideana es muy remota en esa teoría, pero no impide que sea posible. Desde el punto de vista de tal teoría, el enunciado (10) es una verdad metafísicamente contingente. Pues bien, considérese qué veredicto a priori hubiesen dado los "sabios" sobre este enunciado en, por ejemplo, el siglo XVII. Parece razonable pensar que este enunciado hubiese sido declarado sencillamente imposible por motivos a priori. En ese entonces no resultaba concebible para los "sabios" que hubiese geometrías no euclideanas y mucho menos resultaba concebible que la estructura del espacio-tiempo quedase reflejada por alguna geometría no euclideana. Faltaban algunos siglos de desarrollo de las ciencias formales y empíricas para que (10) llegase a ser concebible. Por esa época, sin perjuicio de nuestras limitaciones cognitivas, seguía siendo un rasgo contingente del mundo donde los "sabios" habitaban el que la geometría del espacio-tiempo era curva.

La maniobra obvia que podría intentar el defensor del principio (3), esto es, de la identificación entre posibilidad metafísica y epistémica, es que una reflexión racional idealizada hubiese debido llegar al veredicto de que (10) es posible, pero esto nos arroja de nuevo al 
problema de precisar qué ha de contar como una reflexión racional idealizada. ¿Quiere decir que los "sabios" del siglo XVII no consideraban posible (10) porque cometían errores de cálculo, y tenían olvidos y desatenciones? No lo creo. Cualquiera que sea el defecto cognitivo que se le pueda enrostrar a Newton, por ejemplo, no parece justo afirmar que hacía mal sus cálculos. Por lo tanto, ¿acaso se quiere decir que los "sabios" tenían capacidades cognitivas reducidas, pero que si éstas fuesen extendidas entonces se llegaría al veredicto de que (10) es posible? Tampoco esto es muy admisible. Efectivamente, podía determinar a priori que (10) es posible, pero esto no viene a cuento aquí.

En el caso de las necesidades fuertes, Chalmers ha sostenido sencillamente que tales tesis no están — todavía - suficientemente justificadas (por ejemplo, la existencia de Dios o la identidad psicofísica). Esto es bastante débil, por lo pronto, pues si llegásemos a poseer en el futuro mayor claridad sobre estas cuestiones, la maniobra defensiva de Chalmers quedaría totalmente inservible. En todo caso, lo que en este artículo se ha mostrado es que Chalmers ha desatendido completamente la otra vertiente del problema: la existencia de contingencias fuertes en que las maniobras defensivas son muchísimo más difíciles. En efecto, si en el caso de las necesidades fuertes Chalmers ha sostenido que un buen motivo para pensar que los enunciados en cuestión no son, después de todo, necesarios, es que son epistémicamente contingentes; parece difícil que en los casos de contingencias fuertes se sostenga que, después de todo, no son enunciados contingentes ya que son epistémicamente imposibles. ¿Va Chalmers a sostener que era realmente imposible una estructura geométrica curva del espaciotiempo en el siglo XVII? Como se ve, ésta es un área en la que las dificultades para el proyecto ambicioso de Chalmers y Jackson son muchísimo mayores.

\section{Conclusiones}

El esquema bidimensional epistémico está asociado a un programa filosófico sumamente ambicioso en el que se pretende reivindicar la relevancia del análisis conceptual a priori para resolver las cuestiones metafísicas más fundamentales y, en particular, en el que se pretende sustituir la noción de modalidad metafísica por la de modalidad epistémica. En principio, el esquema bidimensional epistémico vendría a aliviar las dificultades que encuentra usualmente este programa ambicioso. 
El examen de la cuestión ha mostrado que subsisten muchas dificultades en el esquema propuesto, tres de las cuales han sido presentadas en este trabajo. En primer lugar, no se ha logrado diferenciar con claridad el bidimensionalismo epistémico de las formas contextuales tradicionales. En segundo lugar, todo el espacio modal epistémico y las intensiones primarias y secundarias definidas sobre él, están dependiendo de la noción de justificación a priori (reflexión racional idealizada). Esta noción, sin embargo, necesita urgentemente mayor precisión. En tercer lugar, la conexión crucial entre modalidad epistémica y modalidad metafísica no está justificada. Chalmers sólo ha dado indicaciones programáticas sobre la cuestión, basado en el hecho de que no se han presentado todavía argumentos suficientemente contundentes a favor de "necesidades fuertes". Se han desatendido completamente, sin embargo, los casos de "contingencias fuertes", las cuales plantean un problema aún más formidable para el programa bidimensional.

Puede verse, por lo tanto, que todavía queda un camino arduo y largo por recorrer para el desarrollo de las tesis características del bidimensionalismo más ambicioso. Las dificultades que se han presentado aquí no deben verse, necesariamente, como objeciones definitivas, pero está claro que hay muchas cosas que requieren todavía una explicación satisfactoria. Mientras no se den esas explicaciones, lo más razonable parece simplemente quedarse con la distinción entre modalidades metafísicas y conceptos epistemológicos enseñada por Kripke. La conexión áurea no ha sido restaurada, al menos, no todavía. ${ }^{8}$

\section{BIBLIOGRAFÍA}

Chalmers, D., 2006, "The Foundations of Two-Dimensional Semantics", en M. García-Carpintero y J. Maciá (comps.), Two-Dimensional Semantics: Foundations and Applications, Oxford University Press, Oxford, pp. 55140.

__ 2002, “Does Conceivability Entail Possibility?”, en T. Szabó Gendler y J. Hawthorne (comps.), Conceivability and Possibility, Clarendon Press, Oxford, pp. 145-200.

- 1996, The Conscious Mind: In Search of a Fundamental Theory, Oxford University Press, Oxford. [Versión en castellano: La mente

${ }^{8}$ La redacción de este trabajo se realizó en el marco del proyecto de investigación FONDECYT 1070339 (CONICYT, Chile). Agradezco las sugerencias de un dictaminador anónimo de esta revista para mejorar la versión final. 
consciente. En busca de una teoría fundamental, trad. José A. Álvarez, Gedisa, Barcelona, 1999.]

Jackson, F., 1998, From Metaphysics to Ethics. A Defence of Conceptual Analysis, Clarendon Press, Oxford.

Kaplan, D., 1990, “Thoughts on Demonstratives", en P. Yourgrau (comp.), Demonstratives, Oxford University Press, Oxford, pp. 34-49.

Kripke, S., 1980, Naming and Necessity, Blackwell, Oxford. [Versión en castellano: El nombrar y la necesidad, 2a. ed., trad. Margarita M. Valdés, Instituto de Investigaciones Filosóficas-UNAM, México, 1995.]

Putnam, H., 1981, Reason, Truth, and History, Cambridge University Press, Cambridge. [Versión en castellano: Razón, verdad e historia, trad. José Miguel Esteban, Tecnos, Madrid, 1988.]

Soames, S., 2005, Reference and Description. The Case Against TwoDimensionalism, Princeton University Press, Princeton.

Recibido el 26 de diciembre de 2006; revisado el 10 de septiembre de 2007; aceptado el 23 de octubre de 2007. 